\title{
The case of a southern European glacier disappearing under recent warming that survived Roman and Medieval warm periods
}

\section{Electronic Supplementary Material}

The depth age model was created using the package CLAM 2.2 (Blaauw, 2010; Blaauw et al., 2019) run in $\mathrm{R}$ (R Development Core, 2018 ). CLAM creates depth-age models that can be based on linear interpolation, linear or polynomial regression and cubic, smooth or locally weighted splines. CLAM enables that for each date the probability of a calendar year being sampled is proportionate to its calibrated probability (Blaauw, 2010). Uncertainty ranges as well as a most-likely age-model are produced as a CLAM output.

In order to reproduce the presented age model we provide here details on the chosen parameters.

The dataset to build this age model is available in the file paleoice.csv and a summary of the parameter used are in paleoice_polyn_regr_settings.txt. We chose to perform a linear regression (polynomial of order 1) as the more realistic option to enhance the goodness of fit of our model,with a confidence interval of 0.95 where 1000 iterations were run. The regression was weighted according to the calibrated probabilities of the dates and the age-depth model derived ages averages were weighted too. We set a hiatus at $2700 \mathrm{~cm}$ as explained in the text (equivalent to $73 \mathrm{~m}$ depth following a bottom-up sampling strategy).

The following $\mathrm{R}$ code lines enable creating the current age-depth model. We encourage the reader to read Blaauw (2010) and the online version of CLAM 2.2 before running this code.

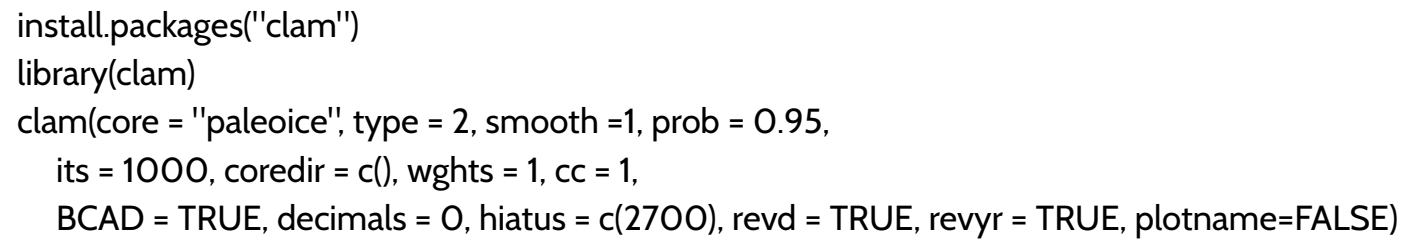

\section{References}

Blaauw, M., 2010. Methods and code for 'classical' age-modelling of radiocarbon sequences. Quaternary Geochronology 5, 512-518. https://doi.org/10.1016/i.quageo.2010.01.002

Blaauw, M., Christen, J.A., Vazquez, J.E., Goring, S., 2019. clam: Classical Age-Depth Modelling of Cores from Deposits. CRAN 2019 https://CRAN.R-project.org/package=clam 